\title{
Neck pain: What if it is not musculoskeletal?
}

Nirosen Vijiaratnam, David R Williams, Kelly L Bertram

\section{Background}

Neck pain is a common presentation in general practice, with muscle strain or osteoarthritis the most common diagnoses. A systematic approach for identifying red flags for alternative causes is required to appropriately investigate or refer for specialist opinion.

\section{Objectives}

The aim of this article is to highlight features of neurological and other causes of neck pain in adults that may present in general practice, and to outline a quick and practical diagnostic approach.

\section{Discussion}

Neck pain in adults may result from musculoskeletal or neurological disease, or as a component of a wide variety of metabolic, infective or malignant disorders. Focused attention to those components of history and examination that suggest alternative conditions can assist the diagnostic process.
NECK PAIN is a leading cause of disability in adults, ${ }^{1}$ reported in up to $20 \%$ of adults, ${ }^{2,3}$ with many presenting for assessment. ${ }^{3}$ One study estimated that general practitioners (GPs) were consulted seven times per week for neck pain. ${ }^{4}$ While the majority of cases are attributable to benign musculoskeletal conditions, neurological and orthopaedic pathology may present similarly. Identifying patients who require investigation and referral involves an understanding of possibilities and clues. These clues need to be elicited in a timely manner owing to the nature of general practice. We outline an approach to alert clinicians regarding potentially serious conditions in adult patients.

\section{Background considerations}

The onset, distribution, nature of pain and associated features guide the approach. Neck pain is typically somatic, neuropathic or both. Somatic pain can be either superficial or deep. Superficial pain is activated by nociceptors, and is typically sharp and distinctly localised. Deep pain is dull and poorly localised, and suggests involvement of deeper structures. Neuropathic pain is caused by irritation of nerve roots (radicular) or the spinal cord. It is sharp or burning in nature, manifests with a combination of neck pain (79\%), pain shooting down the arm in a dermatomal distribution (99\%) or pain in the scapular/parascapular regions (52\%). Associated dysesthesia, paraesthesia or allodynia (85\%) commonly occurs..$^{5-7}$

Consultations occurred between the authors considering potential diagnoses for neck pain. A literature review was subsequently conducted on PubMed with keywords that arose, including the terms 'neck pain' and 'diagnosis'. Studies obtained were assessed for quality and relevance, as were references from studies. Red flags were delineated and a stepwise approach then devised. The final list referenced were pertinent to support the three-cardinal-question approach arrived at by the authors.

\section{Short session, quick action}

The three-cardinal-question approach (Figure 1) was the outcome of the process described. The aim is to address neck pain with three quick questions and identify distinguishing causative features that prompt further attention with varying urgency. Using this approach, the priority is to consider if:

1. the pain may represent a medical emergency

2. the pain requires imaging or further investigations

3. specialist input is necessary.

\section{Is this an emergency?}

\section{Cervical cord compromise}

Sudden-onset neck pain is a hallmark of underlying pathology requiring urgent attention. Following trauma, this can indicate bone fractures or disc lesions. Pain is typically posterior paramedian, with radiation to the occipital and parascapular regions. ${ }^{8}$ Associated neurological deficits can occur occasionally, depending on underlying structural injury. Some suggestive features of structures involved include severe axial rather than extremity pain in disc disease, midline involvement in facet joint pain and specific points of trigger in myofascial pain. ${ }^{8}$ In cervical myelopathy, pain is typically deep and aching, with coexistent radicular features occurring in one-third of presentations. ${ }^{5} \mathrm{~A}$ history of insidious limb or gait dysfunction and incontinence should be sought. Lhermitte's 
phenomenon, an electrical sensation in the back or limbs on flexing the neck, is suggestive but broadly implies an intrinsic lesion of the upper cervical cord or lower brainstem. ${ }^{9}$ Apart from an acute vertebral fracture, other causes include atlantoaxial subluxation in poorly controlled chronic rheumatoid arteritis, ${ }^{10}$ or an expanding epidural mass, such as a tumour, abscess or haemorrhage. ${ }^{11,12}$ Imminent cord compression is a neurosurgical emergency, requiring urgent attention..$^{6,7,13-15}$

Localised tenderness over a vertebral body can suggest local infection in the bone or disc. ${ }^{12}$ Accompanying fever and severe, acute posterior neck pain with restricted neck movement are presenting features of an extradural abscess.

- Cervical cord compromise

- Acute onset with trauma

- Lhermitte's phenomenon ${ }^{9}$

- Progressive limb weakness (non-myotomal)6,7

- Sensory abnormality (non-dermatomal)6,7

- Progressive gait impairment ${ }^{11-14}$

- Bladder and bowel dysfunction ${ }^{11-14}$

Is this an emergency?

\section{- Infection}

- Posterior pain with associated fevers and neck stiffness ${ }^{9}$

- Vascular emergencies

- Sudden onset tearing nature, recurrent thunderclap headache, unilateral weakness, vertigo $^{16-19}$

- Myocardial ischemia

- Typically associated with other cardiac features ${ }^{21}$

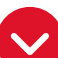

\section{Does this} require further investigation?

- Systemic symptoms, fevers, palpable masses

- Tumours/lesions, radiculopathy

- Radicular pain/sensory abnormality,7

\section{- Neurologist}

- Neck deformity or change in posture 25-30

- Tremor or jerking (head/limbs) ${ }^{32}$

- Neck weakness ${ }^{25}$

- Autonomic symptoms ${ }^{25}$

Does this
require
specialist
input?

- Rheumatologist

- Early morning stiffness ${ }^{10}$

- Associated limb girdle pain/entire back involvement/joint pains or deformity/ tendonitis ${ }^{10}$

\section{- Neurosurgeon}

- Radiculopathy with imaging correlating ${ }^{23}$

Figure 1. Three-cardinal-question approach to neck pain

Furthermore, extensive neurological signs outside a single dermatome or myotome must prompt complete neurological assessment of all limbs to pursue urgent imaging. A plain X-ray is insufficient and magnetic resonance imaging (MRI) must be performed. ${ }^{13}$

\section{Infection}

Meningitis also causes posterior pain and neck rigidity, with a rapid decline in conscious state, and requires urgent antibiotics. ${ }^{9}$ The presence of painful nuchal rigidity, or Kernig's sign, must prompt further investigation for infection. ${ }^{9}$ If meningitis is suspected, an urgent lumbar puncture for microbiological assessment is mandatory and imminent administration of antibiotics warranted. ${ }^{9}$

\section{Vascular emergencies}

Sudden-onset, unilateral 'tearing' neck pain, with unilateral headache and associated vertigo, makes a vascular dissection highly probable. ${ }^{16}$ A more indolent course and non-localised pain does not, however, exclude this diagnosis; in particular, carotid dissection can present without initial neurological deficits. ${ }^{17-19}$ Acute-onset limb weakness suggests stroke. Weakness in the case of stroke involves more than one myotome, with extension weakness in the upper limb and flexion weakness in the lower limb predominating. Kernig's sign can be positive with coexistent subarachnoid haemorrhage. ${ }^{18,19}$ These findings require immediate referral to a stroke service for urgent brain and vascular imaging.

A history of recurrent thunderclap headache (severe pain peaking within seconds), typically occipital and predating sudden posterior neck pain, is highly suggestive of reversible cerebral vasoconstriction syndrome (RCVS). Neck pain occurs in $15 \%$ of RCVS cases and should alert investigation for complicating cervical arterial dissection. ${ }^{20}$ This syndrome also requires urgent attention for immediate imaging.

Although atypical, neck pain can be a feature of myocardial ischemia. This symptom, however, almost never occurs in isolation and usually accompanies more typical cardiac ischaemic features. ${ }^{21}$ 
When it occurs, occipital and posterior neck regions predominate (in over 50\% of cases), while head (43.3\%) and anterior neck (43.3\%) involvement can occur less frequently. ${ }^{21}$ This is an emergency and requires immediate cardiac evaluation.

\section{Does this require further investigation?}

A number of coexistent features occurring with neck pain and examination findings should prompt further investigation for a spectrum of conditions.

\section{Limb weakness}

Disc protrusion or extrusion causing apparent nerve compression on imaging may cause acute severe pain in the neck or back, with radicular pain, and be accompanied by specific myotomal weakness and segmental sensory change. ${ }^{5}$ A Spurling manoeuver (axial loading of the neck while the head is extended or rotated) or a shoulder abduction and upper limb tension test help predict cervical radiculopathy ${ }^{22,23}$ and distinguish from shoulder pathology. Examination findings include dermatomal sensory change, myotomal weakness and tendon reflex abnormality. Focal tenderness in the neck is usually concordant with the site of local or somatic referred pain (eg C2-3 facet joint, right upper neck pain and headache). ${ }^{24}$ Imaging can confirm the diagnosis, with electromyography determining the severity of nerve injury and appropriateness of surgery. Neurosurgical input is warranted in interpreting isolated pain in the absence of neurological cause. Imaging findings of spinal degeneration are common in asymptomatic people. ${ }^{23}$

\section{Fever}

Upper respiratory tract infection can manifest with anterior neck compartment pain and produce palpable lymphadenopathy. Quinsy, or peritonsillar abscess, produces an alteration to the quality of speech, odynophagia and, potentially, breathing difficulty. The latter findings may occasionally require further imaging, typically a computed tomography scan to exclude impending airway compression or consideration for surgical drainage.

\section{Neck swelling}

Swelling in the anterior neck is likely to be either infectious or malignant, and careful examination should determine the affected organ. Thyroiditis may produce rapid swelling of the thyroid gland and anterior compartment pain. This may be accompanied by other systemic signs of hormonal dysfunction. Ultrasound examination can help characterise focal thyroid lesions, cystic structures and reactive lymph nodes, while radio-iodine studies after expert endocrine input will demonstrate avidity and imply aetiology. Blood tests for thyroid function, thyroid antibodies and blood counts, including a blood film, may help determine the aetiology and urgency of referral.

\section{Does this require specialist input?}

Coexistent findings outlined below are red flags for neurological or rheumatological conditions that should prompt referral. Overarching features include early morning stiffness and joint, skin and nail changes in rheumatalogical conditions; and altered neck posture, tremor, limb stiffness and autonomic symptoms in neurological conditions.

\section{Neck stiffness}

Early morning stiffness that improves through the day is indicative of rheumatological conditions (rheumatoid arthritis, ankylosing spondylitis, myositis, diffuse idiopathic skeletal hyperostosis, polymyalgia rheumatic and fibromyalgia), but these do not usually occur in isolation. ${ }^{10}$ Generalised joint involvement and skin changes typically coexist..$^{10}$ Although muscular strains are more common, they typically do not affect range of motion and are accompanied by cervicogenic headaches. Stiffness also occurs with Parkinsonism, accompanied by limb symptoms. Accompanying reduced motion range or abnormal neck posture are features of temporary torticollis or spasmodic torticollis.

\section{Abnormal neck posture}

Abnormal neck posture occurs with a neck mass or bone changes (osteoporotic crush fractures, ankylosing spondylitis and joint dislocations) producing a deformity.
Muscle disease or myasthenia, producing posterior neck muscle weakness, may present with neck discomfort from forward head drooping. ${ }^{25}$ Head drop occurs in multiple system atrophy; autonomic symptoms and marked postural hypotension coexist. ${ }^{25}$ In stiff person syndrome and tetany, abnormal neck extension occurs with stiffness throughout the spine..$^{25}$ Cervical dystonia is characterised by involuntary movements and sustained contractions of neck and shoulder muscles. ${ }^{26,27}$ Neck pain can be the presenting symptom and occurs in $80 \%$ of cases, often radiating to the shoulder, arm and torso. ${ }^{28-31}$ On examination, neck posture varies, in particular with walking or movement. In contrast, muscular or neuromuscular diseases produce difficulty lifting the head, without variation. Neck flexion in Parkinson's disease may be temporarily alleviated by medication or voluntary action. ${ }^{25}$ Young-onset cervical dystonia may follow neuroleptic medication, but should prompt cerebral MRI and testing for Wilson's disease. In middle age, imaging is usually not required and further neurological input for symptom management sought as appropriate.

\section{Tremor}

Head tremor with neck pain can be induced by cervical dystonia, essential tremor and Parkinson's disease. Dystonic tremors have variable frequency, with irregular amplitude, and vary with activity. Essential tremor is oscillatory and regular, typically also involving the upper limbs in posture and occurring with activities such as carrying objects. Rest tremor is unusual. ${ }^{32}$ Tremor in Parkinson's disease is oscillatory, regular and has a frequency of 4-6 Hz. The tremor may be present at rest, during posture and movement, but does not amplify with posture. Lip or chin tremor is typical, but not head tremor. The presence of bradykinesia in Parkinson's disease is essential for the clinical diagnosis, and a liberal treatment with levodopa should be considered prior to neurological referral.

\section{Conclusion}

Neck pain in adults is a common presentation to general practice, but 
may represent a diverse range of pathology. Components on history and examination with a three-step question approach will guide identification of urgent conditions requiring imaging and immediate treatment, or rare conditions requiring specialist input.

\section{Authors}

Nirosen Vijiaratnam MBBS BMedSci, Neurosciences, Alfred Hospital, Melbourne, Vic

David R Williams MBBS, PhD, FRACP,

Neurosciences, Alfred Hospital, Melbourne, Vic;

Faculty of Medicine Nursing and Health Sciences, Monash University, Melbourne, Vic

Kelly L Bertram MBBS, FRACP, Neurosciences, Alfred Hospital, Melbourne, Vic; Faculty of Medicine Nursing and Health Sciences, Monash University, Melbourne, Vic. k.bertram@alfred.org.au

Competing interests: Dr Vijiaratnam receives unrestricted educational grants from Ipsen and Biogen and has received travel grants from Ipsen, Abbvie, CSL Behring and the Movement Disorder Society. Dr Bertram has received a research grant from the Brain Foundation and Parkinson's Victoria, and has received unrestricted educational grants from Ipsen and Allergan. Dr Williams has an intellectual property right for the Parkinson's Disease Assessment App, receives consultancies from Abbvie, Ipsen and Allergan, is on the advisory boards of Abbvie, Ipsen and Allergan and receives a grant from Abbvie.

Provenance and peer review: Not commissioned, externally peer reviewed.

\section{References}

1. Cohen SP. Epidemiology, diagnosis, and treatment of neck pain. Mayo Clin Proc 2015;90(2):284-99. doi: 10.1016/j.mayocp.2014.09.008.

2. Moradi-Lakeh M, Forouzanfar MH, Vollset SE, et al. Burden of musculoskeletal disorders in the Eastern Mediterranean Region, 1990-2013: Findings from the Global Burden of Disease Study 2013. Ann Rheum Dis 2017;76(8):1365-73. doi: 10.1136/annrheumdis-2016-210146.

3. Woodhouse A, Pape K, Romundstad PR, Vasseljen $\mathrm{O}$. Health care contact following a new incident neck or low back pain episode in the general population; the HUNT study. BMC Health Serv Res 2016;16:81. doi: 10.1186/s12913-016-1326-5.

4. Bot SD, Van der waal JM, Terwee CB, et al. Incidence and prevalence of complaints of the neck and upper extremity in general practice. Ann Rheum Dis 2005:64(1):118-23.

5. Henderson CM, Hennessy RG, Shuey HM, Shackelford EG. Posterior-lateral foraminotomy as an exclusive operative technique for cervical radiculopathy: A review of 846 consecutively operated cases. Neurosurgery 1983;13(5):504-12.

6. Carette S, Fehlings MG. Clinical practice. Cervical radiculopathy. N Eng J Med 2005;353(4):392-99.

7. Bono CM, Ghiselli G, Gilbert TJ, et al. An evidence-based clinical guideline for the diagnosis and treatment of cervical radiculopathy from degenerative disorders. Spine J 2011;11(1):64-72. doi: 10.1016/j.spinee.2010.10.023.

8. Douglass AB, Bope ET. Evaluation and treatment of posterior neck pain in family practice. J Am Board Fam Pract 2004;17 Suppl:S13-22.
9. Durand ML, Calderwood SB, Weber DJ, et al. Acute bacterial meningitis in adults. A review of 493 episodes. N Eng J Med 1993;328(1):21-28.

10. Oberstein EM, Carpintero M, Hopkins A. Neck pain from a rheumatologic perspective. Phys Med Rehabil Clin N Am 2011;22(3):485-502. doi: 10.1016/j.pmr.2011.02.009.

11. Abdu WA, Provencher M. Primary bone and metastatic tumors of the cervical spine. Spine (Phila Pa 1976) 1998;23(24):2767-77.

12. Beronius M, Bergman B, Andersson R. Vertebral osteomyelitis in Göteborg, Sweden: A retrospective study of patients during 1990-95. Scand J Infect Dis 2001;33(7):527-32.

13. BMJ Best Practice Guidelines. Neck Pain. UK: BMJ Publishing Group, 2016. Available at http:// bestpractice.bmj.com/topics/en-gb/943/ diagnosis-approach [Accessed 9 April 2018].

14. Lawrence JS. Disc degeneration: Its frequency and relationship to symptoms. Ann Rheum Dis 1969;28(2):121-38.

15. Darouiche RO. Spinal epidural abscess. N Eng J Med 2006;355(19):2012-20.

16. Fukuhara K, Ogata T, Ouma S, et al. Impact of initial symptom for accurate diagnosis of vertebral artery dissection. Int J Stroke 2015;10 Suppl A100:30-33. doi: 10.1111/ijs.12546.

17. Traenka C, Dougoud D, Simonetti BG, et al. Cervical artery dissection in patients $\geq 60$ years: Often painless, few mechanical triggers. Neurology 2017;88(14):1313-20. doi: 10.1212/ WNL.0000000000003788.

18. Boyle E, Côté P, Grier AR, Cassidy JD. Examining vertebrobasilar artery stroke in two Canadian provinces. Spine (Phila Pa 1976) 2008;33(Suppl):S170-75. doi: 10.1097/ BRS.0b013e31816454e0.

19. Cassidy JD, Boyle E, Côté P, et al. Risk of vertebrobasilar stroke and chiropractic care: Results of a population-based case-control and case-crossover study. Spine (Phila $\mathrm{Pa}$ 1976) 2008;33(4 Suppl):S176-83. doi: 10.1097/ BRS.0b013e3181644600.

20. Mawet J, Boukobza M, Franc J, et al. Reversible cerebral vasoconstriction syndrome and cervical artery dissection in 20 patients. Neurology 2013;81(9):821-24. doi: 10.1212/ WNL.0b013e3182a2cbe2.

21. Bakhshi M, Rezaei R, Baharvand M, Bakhtiari S. Frequency of craniofacial pain in patients with ischemic heart disease. J Clin Exp Dent 2017;9(1):e91-95. doi: 10.4317/jced.53078.

22. Thoomes EJ, van Geest S, van der Windt DA, et al. Value of physical tests in diagnosing cervical radiculopathy: A systematic review. Spine J 2018;18(1):179-89. doi: 10.1016/j. spinee.2017.08.241.

23. Wainner RS, Fritz JM, Irrgang JJ, Boninger ML, Delitto A, Allison S. Reliability and diagnostic accuracy of the clinical examination and patient self-report measures for cervical radiculopathy. Spine (Phila Pa 1976) 2003;28(1):52-62.

24. Tanaka Y, Kokubun S, Sato T, Ozawa H. Cervical roots as origin of pain in the neck or scapular regions. Spine (Phila Pa 1976) 2006;31(17):E568-73.

25. Umapathi T, Chaudhry V, Cornblath $D_{\text {, }}$ Drachman D, Griffin J, Kuncl R. Head drop and camptocormia. J Neurol Neurosurg Psychiatry 2002;73(1):1-7.

26. Albanese A, Bhatia K, Bressman SB, et al. Phenomenology and classification of dystonia: A consensus update. Mov Disord 2013;28(7):863-73. doi: $10.1002 /$ mds. 25475 .
27. Albanese A. The clinical expression of primary dystonia. J Neurol 2003;250(10):1145-51.

28. Bertram KI, Williams DR. Delays to the diagnosis of cervical dystonia. J Clin Neurosci 2016:25;6264. doi: 10.1016/j.jocn.2015.05.054.

29. Chan J, Brin MF, Fahn S. Idiopathic cervical dystonia: Clinical characteristics. Mov Disord 1991;6(2):119-26.

30. Werle RW, Takeda SY, Zonta MB, Guimarães AT, Teive HA. The physical, social and emotional aspects are the most affected in the quality of life of the patients with cervical dystonia. Arq Neuropsiquiatr 2014;72(6):405-10.

31. Kutvonen $O$, Dastidar P, Nurmikko T. Pain in spasmodic torticollis. Pain 1997;69(3):279-86.

32. Louis ED, Hernandez N, Michalec M. Prevalence and correlates of rest tremor in essential tremor: Cross-sectional survey of 831 patients across four distinct cohorts. Eur J Neurol 2015;22(6):927-32. doi: 10.1111/ene.12683. 